21st Particles and Nuclei International Conference (PANIC 2017)

International Journal of Modern Physics: Conference Series

Vol. 46 (2018) 1860044 (7 pages)

(C) The Author(s)

DOI: $10.1142 / \mathrm{S} 2010194518600443$

\title{
Measurements of the Reactor Antineutrino with Solid State Scintillation Detector
}

\author{
I. Alekseev ${ }^{1,2,3, *}$, V. Belov 4 , V. Brudanin ${ }^{4}$, M. Danilov ${ }^{2,3,5}$, V. Egorov ${ }^{4,6}$, D. Filosofov ${ }^{4}$, \\ M. Fomina ${ }^{4}$, Z. Hons ${ }^{4,7}$, S. Kazartsev ${ }^{4,6}$, A. Kobyakin ${ }^{1,3}$, A. Kuznetsov ${ }^{4}$, I. Machikhiliyan ${ }^{1,2}$, \\ D. Medvedev 4 , V. Nesterov ${ }^{1}$, A. Olshevsky ${ }^{4}$, N. Pogorelov ${ }^{1}$, D. Ponomarev ${ }^{4}$, I. Rozova ${ }^{4}$, \\ N. Rumyantseva ${ }^{4}$, V. Rusinov ${ }^{1}$, A. Salamatin ${ }^{4}$, E. Samigullin ${ }^{1,2}$, Ye. Shevchik ${ }^{4}$, M. Shirchenko ${ }^{4}$, \\ Yu. Shitov ${ }^{4,8}$, N. Skrobova ${ }^{1,3,5}$, A. Starostin ${ }^{1,2}$, D. Svirida ${ }^{1,2}$, E. Tarkovsky ${ }^{1}$, I. Tikhomirov ${ }^{1}$, \\ J. Vlášek ${ }^{4,9}$, I. Zhitnikov ${ }^{4}$, D. Zinatulina ${ }^{4}$ \\ 1 Alikhanov Institute for Theoretical and Experimental Physics, \\ B. Cheremushkinskaya 25, Moscow, 117218, Russia \\ 2 National Research Nuclear University MEPhI (Moscow Engineering Physics Institute), \\ Kashirskoe highway 31, Moscow, 115409, Russia \\ 3 Moscow Institute of Physics and Technology, \\ 9 Institutskiy per., Dolgoprudny, Moscow Region, 141701, Russia \\ 4 Joint Institute for Nuclear Research, \\ Joliot-Curie 6, Dubna, Moscow region, 141980, Russia \\ 5 Lebedev Physical Institute of the Russian Academy of Sciences, \\ 53 Leninskiy Prospekt, Moscow, 119991, Russia \\ 6 Dubna State University, \\ Universitetskaya 19, Dubna, Moscow Region, 141982, Russia \\ 7 Nuclear Physics Institute, \\ Řez 130, 25068 Řě̃, Cz, Czech Republic \\ 8 Imperial College London, \\ South Kensington Campus, SW7 2AZ, London, United Kingdom \\ ${ }^{9}$ Czech Technical University in Prague, \\ Zikova 1903/4, 16636 Prague 6, Czech Republic \\ *igor.alekseev@itep.ru
}

Published 3 May 2018

\begin{abstract}
Measurements of reactor antineutrino play an important role in the efforts at the frontier of the modern physics. The DANSS collaboration presents preliminary results of a one year run with a cubic meter solid state detector placed below $3.1 \mathrm{GW}$ industrial light water reactor. The experiment is sensitive to sterile neutrino in the most interesting region of mixing parameter space. 2500 scintillation strips of the sensitive volume of the detector have multilayer passive shielding of copper, lead and borated polyethylene and active muon veto. Detector position below the reactor gives an advantage of overburden about $50 \mathrm{~m}$ of water equivalent providing factor of six in cosmic muon suppression and eliminating fast neutrons. The detector is placed on a vertically movable platform which
\end{abstract}

This is an Open Access article published by World Scientific Publishing Company. It is distributed under the terms of the Creative Commons Attribution 4.0 (CC-BY) License. Further distribution of this work is permitted, provided the original work is properly cited. 


\section{Alekseev et al.}

allows to change the distance to the reactor core center in the range 10.7-12.7 $\mathrm{m}$ within a few minutes. The strips are read out individually by SiPMs and in groups of 50 by PMTs. 5000 inverse beta-decay events per day are collected in the fiducial volume, which is $78 \%$ of the whole detector, at the position closest to the reactor. Overburden, active veto and good segmentation of the detector result in an excellent signal to background ratio.

The talk is dedicated to the data analysis and preliminary results. The experiment status is also presented.

Keywords: Reactor antineutrinos; inverse beta-decay; neutrino oscillations; sterile neutrino.

\section{Introduction}

Great progress of neutrino measurements during last 20 years established an existence of oscillations between three neutrino flavors. Many oscillation parameters are now measured with a good precision, though the question of neutrino mass hierarchy is still open. So neutrino studies are still promising new physics ${ }^{1}$. Analysis of the existing reactor data by $\mathrm{G}$. Mention et al. ${ }^{2}$ demonstrated that it can be explained by introduction of a new type of neutrino with parameters $\Delta m_{\text {new }}^{2}>1.5 \mathrm{eV}^{2}$ and $\sin ^{2}\left(2 \theta_{\text {new }}\right)=0.14 \pm 0.08$. These new neutrinos do not interact with usual matter but are seen in the disappearance of the usual neutrinos via oscillations:

$$
P(L)=1-\sin ^{2}\left(2 \theta_{\text {new }}\right) \sin ^{2}\left(1.27 \frac{\Delta m_{\text {new }}^{2}\left[e V^{2}\right] L[m]}{E_{\nu}[M e V]}\right),
$$

where $P(L)$ is the probability of the original neutrino to survive, $E_{\nu}$ - its energy and $L$ is the distance from the neutrino source. The idea of DANSS experiment is to measure neutrino spectrum at different distances with the same detector ${ }^{3}$, which allows to cancel all systematic errors arising from comparison of spectra obtained by different detectors. An illustration of the idea is given in Fig. 1, where ratio of spectra at two detector positions from the center of the reactor core $(10.7 \mathrm{~m}(\mathrm{U})$ and $12.7 \mathrm{~m}(\mathrm{D}))$ is shown for three values of $\Delta m_{\text {new }}^{2}$. This figure does not include smearing by the reactor and detector sizes as well as the detector resolution.

Detection of the reactor antineutrino is based on the Inverse Beta-Decay (IBD) reaction: $\bar{\nu}_{e}+p \rightarrow e^{+}+n$. This process produces signals, separated by time needed

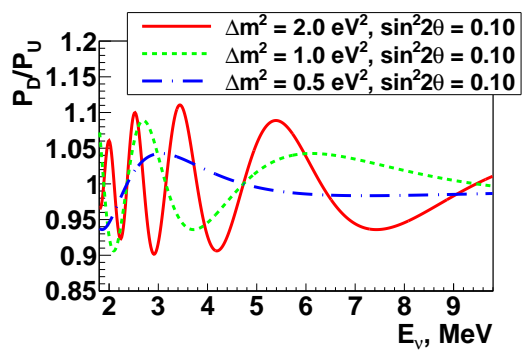

Fig. 1. Naïve ratio of the spectra at the farthest and the closest detector positions for different $\Delta m_{\text {new }}^{2}$. 
for neutron moderation and capture. The first signal, coming immediately from positron track ionization and annihilation is usually called "prompt". The other signal from neutron capture is called "delayed". The time difference between the prompt and the delayed signal is in the tens of microseconds range, providing a very good reaction signature. For reactor antineutrino energy of the recoiled neutron is small, so the positron takes the original neutrino energy with the subtraction of $1.804 \mathrm{MeV}$ - the reaction threshold. The neutron capture by gadolinium produces a flash of $\gamma$-rays with the total energy about $8 \mathrm{MeV}$.

\section{Experiment}

The DANSS detector is a cubic meter of plastic scintillator surrounded by multilayer passive and active shielding ${ }^{4}$. The scintillator is arranged as 2500 strips $(1 \times 4 \times$ $\left.100 \mathrm{~cm}^{3}\right)$. Gadolinium is added to the outer $0.2 \mathrm{~mm}$ thick white layer covering strips. Total amount of gadolinium is $0.35 \%$ by weight. Each strip has three wavelength shifting fibers (Kuraray Y-11, $\emptyset 1.2 \mathrm{~mm}$ ) glued in groves on its upper surface. The center fibers are used for individual SiPM (Hamamatsu MPPC S12825-050C(X)) readout, providing fine space resolution. Two edge fibers from a group of 50 strips are bundled together to a conventional PMT (Hamamatsu R7600U-300) to be used in trigger. Passive shielding includes $5 \mathrm{~cm}$ of copper, $8 \mathrm{~cm}$ of borated polyethylene, $5 \mathrm{~cm}$ of lead and $8 \mathrm{~cm}$ of borated polyethylene. Five sides of the cube, with the exception of the bottom, are covered by two layers of $3 \mathrm{~cm}$ thick scintillator counters forming an active shielding.

The experiment is situated in a room just below $3.1 \mathrm{GW}$ industrial light water reactor of Kalininskaya nuclear power plant (KNPP). This position provides both advantages of high neutrino flux $\left(\sim 5 \cdot 10^{13} \mathrm{~cm}^{-2} \mathrm{~s}^{-1}\right.$ in the closest detector position) and $50 \mathrm{~m}$ of water equivalent overburden. The detector is placed on the platform movable vertically, which allows to change distance between reactor core and the detector in the range $10.7-12.7 \mathrm{~m}$ (center to center). A weekly detector motion cycle with three positions 10.7 (up), 11.7 (middle) and $12.7 \mathrm{~m}$ (down) was used during data taking.

A dead-time-less DAQ system of DANSS is based on specially designed waveform digitizer modules ${ }^{5}$. Trigger is issued when either energy deposit in 50 PMTs of the detector exceeds $0.7 \mathrm{MeV}$ or energy deposit in active shielding (veto) counters exceeds $3 \mathrm{MeV} .512$ ns waveforms of all channels hit are stored for off-line analysis. For the purpose of SiPM continuous calibration SiPM noise signals are written with large prescale independently from common trigger. Energy calibration of the detector was done using signals from cosmic muons.

\section{Analysis}

Data treatment starts with waveform analysis, SiPM and PMT calibration ${ }^{6}$. Then SiPM hits are filtered in order to reduce number of noise hits in the final data sample. For this purpose average fine trigger time is found, giving more weight to 
more energetic hits. SiPM hits out of \pm 15 ns time window are dropped. Single pixel $\mathrm{SiPM}$ hits require confirmation in the corresponding PMT. On the next step for each trigger its parameters are calculated including a search for continuous ionization cluster, considered as positron candidate. Both SiPM and PMT energy information is used. We consider a trigger as a muon background event if two or more veto counters hit, or energy in a single veto counter is above $4 \mathrm{MeV}$ or energy in the DANSS core is above $20 \mathrm{MeV}$. IBD event selection is based on time correlation between two triggers. Here is the list of cuts applied:

(1) time between prompt and delayed events is in the range $(2-50)$ us;

(2) total energy of the delayed event is in the range $(3.5-15) \mathrm{MeV}$;

(3) there are four or more SiPM hits in the delayed event;

(4) energy of the ionization cluster of the prompt event is in the range $(1-20) \mathrm{MeV}$;

(5) the prompt event energy out of the ionization cluster is less than $1.8 \mathrm{MeV}$;

(6) number of SiPM hits in the prompt event out of the ionization cluster is less than 11 ;

(7) the most energetic hit in the prompt event out of the ionization cluster is less than $0.8 \mathrm{MeV}$;

(8) the ionization cluster of the prompt event is at least $4 \mathrm{~cm}$ from the edge;

(9) vertical distance between the ionization cluster of the prompt event and average coordinate of the delayed event is less than $40 \mathrm{~cm}$;

(10) distance between the ionization cluster of the prompt event and average coordinate of the delayed event is less than $55 \mathrm{~cm}$ in case when all three coordinates are known and less than $45 \mathrm{~cm}$ in proper plane when we know only two coordinates of the positron;

(11) no muon event at least 60 us before the prompt event;

(12) no any triggers with the exception of the delayed event in the time range 45 us before and 80 us after the prompt event;

(13) no triggers with total energy deposit more than $300 \mathrm{MeV} 200$ us before the prompt event.

Cuts (1), (9) and (10) establish correlation between prompt and delay events. Cuts (2)-(7) refine selection of prompt and delay candidates. Cut (8) defines fiducial volume stepping $4 \mathrm{~cm}$ from the scintillation cube edges, because neutron detection efficiency significantly drops close to the edge. On the other hand, background level close to the cube surface is higher. Cuts (11)-(13) are aimed to suppress background due to cosmic muons.

For each physical distribution subtraction of uncorrelated background was done using the same data sample. For this purpose prompt events are searched in sixteen 50 us time windows moved backward for 5, 10, 15, etc. ms. All selection criteria are applied. An example of this approach is presented in Fig. 2, where time difference between prompt and delayed events is shown. Two sources of correlated background were found essential and also subtracted. One is connected to the untagged muon events because of some transparency of veto system. The spectra of these events is 


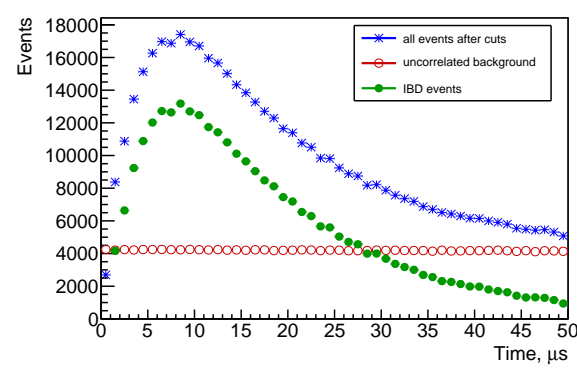

Fig. 2. Time between prompt and delayed events.

assumed the same as of tagged muon events. Its fraction was measured during the reactor partial shutdown in October 2016 to be $5.6 \%$ of tagged muon events (about $2.5 \%$ of IBD events in the detector position closest to the reactor). Another source of correlated background comes from muon induced fast neutrons. This background produces long tail at high positron energies of about $0.1 \%$ of the counting rate at the closest detector position in the region of interest.

\section{Results}

The DANSS position close to the center of the reactor provides a high IBD counting rate, which allows to monitor the reactor power via the antineutrino flux. Figure 3 shows history of DANSS IBD counts in the period since October 2016 till May 2017 compared with standard reactor power measurements in the same periods. Measurements at different detector positions and are corrected as $1 / r^{2}$. Contributions from the neighbor reactors is subtracted. normalization is done using 12 data points in November-December 2012. Measured positron energy is calculated from the energy of the ionization cluster of the prompt event and corrected to average shifts due to energy losses in the strip boundaries and penetration of annihilation energy into cluster. The correction is based on a detailed Monte Carlo simulation of the detector. Resulting spectra for the three detector positions are shown in Fig. 4. An inserted panel in Fig. 4 presents ratio between the farthest and the closest positions. Its fit with a constant has a good $\chi^{2}$, which indicates consistency with 3 neutrino mixing scenario at this level of statistics ${ }^{\mathrm{a}}$. Analysis of exclusion regions based on this ratio by CLs method can be found in Ref. 7 . We are only at the beginning of data taking and analysis now, but the data already allows to exclude a large and most interesting fraction of the sterile neutrino oscillation parameter space. Our plans include getting more statistics and father development of the analysis, simulations and calibration. DANSS is running, so more interesting results are coming soon.

${ }^{a}$ We follow here the material presented at the Conference in early September when only data till middle of May was processed 


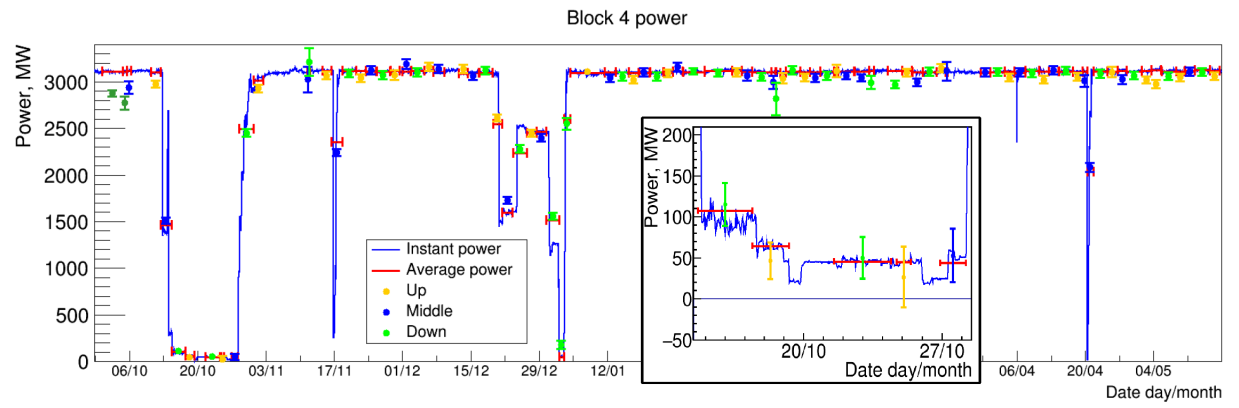

Fig. 3. The reactor power as measured via neutrino counting rate compared with the reactor power measurements neutron fluxes. The insertion gives detailed view of the reactor partial shutdown. Points with error bars correspond to IBD counts at the different detector positions.

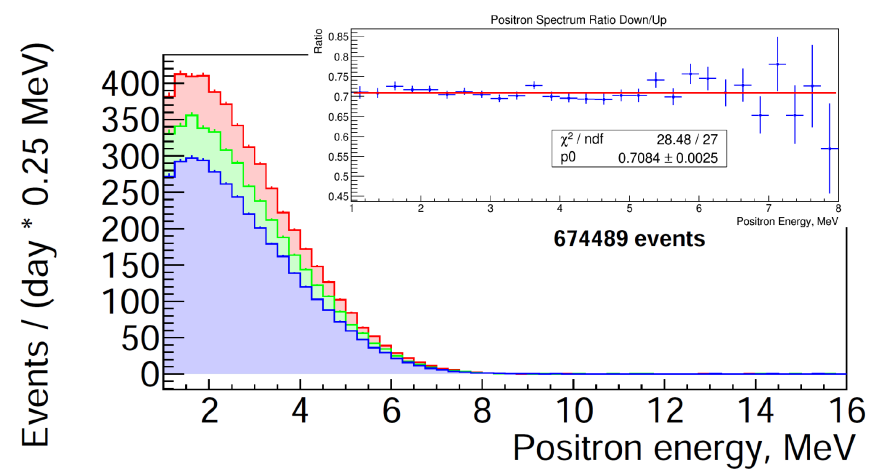

Fig. 4. Distribution over the measured positron energy for three detector positions (up, middle, down) for the data collected from April 2016 till May 2017. Ratio of down to up counts is given in the inserted panel.

\section{Acknowledgments}

The authors are grateful to the directorates of ITEP and JINR for constant support of this work. The authors appreciate the administration of the KNPP and the staff of the KNPP Radiation Safety Department for permanent assistance in the experiment. The detector construction was supported by the Russian State Corporation ROSATOM (state contracts H.4x.44.90.13.1119 and H.4x.44.9B.16.1006) and by the Russian Foundation for Basic Research, grant 09-02-00449. The operation and data analysis became possible due to the valuable support from Russian Science Foundation grant 17-12-01145.

\section{References}

1. K.N. Abazajian et al., Light Sterile Neutrinos: A White Paper, FERMILAB-PUB-12881-PPD (2012); arXiv:hep-ph/1204.5379

2. G. Mention et al. Phys.Rev. D 83073006 (2011); arXiv:hep-ex/1101.2755 
3. M. Danilov (for the DANSS Collaboration), Sensitivity of DANSS detector to short range neutrino oscillations, in PoS EPS-HEP2013 493 (2013); arXiv:physics.ins$\operatorname{det/1311.2777;~arXiv:physics.ins-det/1412.0817~}$

N. Skorobova (for the DANSS Collaboration), Nuclear Physics and Engineering (Rus.) 6 222-227 (2015)

4. DANSS Collaboration (I. Alekseev et al.), Journal of Instrumentation 11, P11011 (2016); arXiv:physics.ins-det/1606.02896

5. I.G. Alekseev et al., Digitization of waveforms from photosensors of DANSS detector, to appear in Instruments and Experimental Techniques

6. I. Alekseev et al., The DANSS neutrino spectrometer: calibration of the detector, stability of the response and light yield studies, to appear in Physics of Particles and Nuclei Letters

7. I.G. Alekseev et al., J. Phys.: Conf. Ser. 934012006 (2017) 